\title{
The Association of Dairy Intake With Metabolic Syndrome and Its Components in Adolescents: Tehran Lipid and Glucose Study
}

\author{
Shirin Ghotboddin Mohammadi ${ }^{1}$; Parvin Mirmiran ${ }^{2,}$; Zahra Bahadoran ${ }^{1}$; Yadollah \\ Mehrabi $^{3}$; Fereidoun Azizi ${ }^{4}$ \\ ${ }_{1}^{1}$ Nutrition and Endocrine Research Center, Research Institute for Endocrine Sciences, Shahid Beheshti University of Medical Sciences, Tehran, IR Iran \\ ${ }_{3}^{2}$ Department of Clinical Nutrition and Dietetics, Faculty of Nutrition Sciences and Food Technology, National Nutrition and Food Technology Research Institute, Tehran, IR Iran \\ ${ }_{4}^{3}$ Department of Epidemiology, Faculty of Health, Shahid Beheshti University of Medical Sciences, Tehran, IR Iran \\ 4 Endocrine Research Center, Research Institute for Endocrine Sciences, Shahid Beheshti University of Medical Sciences, Tehran, IR Iran \\ ${ }^{*}$ Corresponding author: Parvin Mirmiran, Department of Clinical Nutrition and Dietetics, Faculty of Nutrition Sciences and Food Technology, National Nutrition and Food Technol- \\ ogy Research Institute, Tehran, IR Iran. Tel: +98-2122357484, Fax: +98-2122416264; 22402463, E-mail: mirmiran@endocrine.ac.ir
}

Received: November 8,2014; Revised: April 8, 2015; Accepted: April 27, 2015

Background: Epidemiological studies have shown that consumption of dairy product plays an important role in prevention and treatment of Metabolic Syndrome(MetS).

Objectives: The objective of this study was to examine the association of dairy intake with MetS and its components in Tehranian adolescents.

Patients and Methods: In this cross-sectional study, 785 adolescent aged 10 to 19 years, participated from the fourth phase of the Tehran Lipid and Glucose Study. Usual dietary intake was assessed using a valid semi quantitative food frequency questionnaire. Total dairy, low fat and high fat dairy, milk, yoghurt and cheese were evaluated. Assessment of anthropometric, biochemical and blood pressure was performed and MetS was defined according to the de Ferranti criteria.

Results: The mean age of subjects was $14.8 \pm 2.9$ years. The prevalence of MetS was 22.2\% (girls: 19.5\% and boys: 25.2\%). The most prevalent risk factor for MetS in boys was high waist circumference (53.4\%) and among girls was low HDL-C (53.1\%). Energy density and intake of protein, total fat, saturated fat, cholesterol, calcium and phosphor were higher in the highest quartile of dairy consumption than the lowest quartile. After adjustments for confounders, odds ratios with 95\% confidence interval for MetS in the highest quartile of total dairy, low fat dairy, high fat dairy, milk, yoghurt and cheese compared with lowest quartile were respectively 0.97(0.57-1.66), 1.44(0.83-2.49), 0.97 (0.56 - 1.67), 0.70 (0.42 - 1.18), $1.62(0.99-2.64)$ and $0.72(0.44-1.18)$.

Conclusions: Results of this study did not support the hypothesis that dairy products consumption protects against MetS and its components.

Keywords: Dairy Products; Adolescent; Hypertension; Obesity; Dislypidemia

\section{Background}

Metabolic Syndrome (MetS) is a common multifactorial metabolic disorder including insulin resistance, abdominal obesity, dyslipidemia and hypertension, all of which directly promote the development of diabetes and cardiovascular disease $(1,2)$. The prevalence of MetS in Iranian adult population has been reported by $30.1 \%$ (3). A higher prevalence of MetS has also been reported in Tehranian adolescents (10.1\%) compared to American (2\% - 4.9\%) and Canadian adolescents $(7.6 \%)(4,5)$. Several factors have been proposed for development of non-communicable diseases; among the environmental factors sedentary lifestyles and on-going nutrition transition with progressive shift to a westernized diet play an important role in the development of MetS and its related metabolic disorders (6).

Dairy products are main sources of protein, calcium and magnesium and it has been suggested that they may have beneficial effects on regulation of energy and glucose homeostasis and prevention of metabolic disorders, hypertension and cardiovascular diseases (79). Limited data are available regarding the association of dairy consumption as well as various types of dairy products with the risk of MetS and its components, especially in adolescents. Some cohort studies on adolescents reported a significant inverse association between dairy consumption and the risk of MetS and findings from a cross-sectional study on adolescents of 15 to 18 years, indicated that only milk intake was inversely associated with cardiometabolic risk factor $(10,11)$. In contrast, higher intake of dairy products had no significant effect on long-term weight gain and the incidence of MetS in adolescents $(12,13)$.

Copyright (C) 2015, Research Institute For Endocrine Sciences and Iran Endocrine Society. This is an open-access article distributed under the terms of the Creative Commons Attribution-NonCommercial 4.0 International License (http://creativecommons.org/licenses/by-nc/4.0/) which permits copy and redistribute the material just in noncommercial usages, provided the original work is properly cited. 


\section{Objectives}

Despite various types of dairy products traditionally being a main part of Iranian diets, to our best knowledge, no study has been conducted to assess the association of dairy products with MetS and its components in an Iranian population. In this study, we aimed to investigate the association of dairy products (milk, yogurt, cheese) with MetS and its components in adolescents of 10 - 19 years, participants of Tehran Lipid and Glucose Study (2009).

\section{Patients and Methods}

\subsection{Subjects}

The current study was conducted within the framework of Tehran Lipid and Glucose Study (TLGS). Briefly, TLGS is a community-based prospective study conducted to investigate and prevent non-communicable diseases, in a representative sample of residents, aged 10 - 19 years, from district 13 of Tehran, the capital city of Iran. The first phase of the TLGS began in March 1999 and data collection, at three-year intervals, is ongoing (14). In this study, 935 adolescents (10 - 19 years), participated in the fourth phase of TLGS (2009 - 2011), were enrolled. Participants with no completed data on anthropometrics and biochemical measurements $(n=8)$, those who consumed drugs that affect the metabolism of glucose, lipids and blood pressure, such as steroids, non-steroidal anti-inflammatory drugs and thyroid hormones ( $\mathrm{n}$ $=17$ ), those with a history of heart disease $(n=17)$, those with special diets $(n=21)$, boys with energy intake $<800$ or $\geq 5000 \mathrm{kcal} / \mathrm{d}$ and girls with energyintake $<600$ or $\geq$ $4500 \mathrm{kcal} / \mathrm{d}(\mathrm{n}=87)$ were excluded from the final analysis (15).

\subsection{Anthropometric Measurements}

While the subjects were minimally clothed and not wearing shoes, weight was measured using a digital scale (model 707, range 0.1 - $150 \mathrm{~kg}$, Seca, Hamburg, Germany) and recorded to the nearest 100 grams. Height was measured with a tape measure (model 208 Portable Body Meter Measuring Devise; Seca), while the subjects were in a standing position and not wearing shoes and shoulders were in a normal position. BMI (Body Mass Index) was calculated by dividing weight ( $\mathrm{kg}$ ) by the square of height $\left(\mathrm{m}^{2}\right)$. Waist Circumferences (WC) was measured using an inelastic tape measure without any pressure to body surface and recorded to the nearest 1 $\mathrm{cm}$, while the person was wearing a thin dress that did not change the waist size (14).

\subsection{Assessment of Dietary Intake}

Usual dietary intake was assessed using a validate 168-item Food Frequency Questionnaire (FFQ). The FFQ consisted of a list of foods with a standard serving size. Trained dietitians, with at least 5 years of experience in the TLGS survey, asked participants to designate their intake frequency for each food item consumed during the past year on a daily, weekly or monthly basis. Portion sizes of consumed foods reported in household measures were then converted to grams (16).

The validity of food frequency questionnaire was previously evaluated by comparing food groups and nutrient values determined from the questionnaire with values estimated from the average of twelve 24-hour dietary recall surveys; reliability of the FFQ was evaluated in a randomly chosen subgroup of 132 subjects by comparing nutrient consumption ascertained by FFQ responses on two occasions. The intra-class correlation coefficients for dairy products between 2 FFQ were 0.73 and 0.68 for men and women, respectively; correlation coefficients of dairy intake estimated using twelve 24-hour dietary recall and FFQ were 0.89 and 0.61 for men and women, respectively. Complete agreement of diary intake based on twelve 24hour dietary recall and FFQ were acceptable (55 and 56.3\% in men and women, respectively) (17).

Because the iranian food composition table is incomplete and has limited data on nutrient content of raw foods and beverages, to analyze foods and beverages for their energy and nutrient content, we used the US Department of Agriculture FCT $(18,19)$. Dairy products have been defined as low fat milk ( $<2 \%$ fat), high fat milk ( $\geq 2 \%$ fat), cocoa milk, yoghurt, high fat yoghurt, cheese, creamy cheese, buttermilk and kashk. Dietary energy density was calculated as following formula:

(1) Dietary energy density $=\left[\frac{\text { Total energy intake }(\mathrm{kcal} / \mathrm{d})}{\text { Total food intakes }(\mathrm{g} / \mathrm{d})}\right] \times 100$

\subsection{Blood Pressure Measurement}

Measurement of blood pressure in the right arm was performed two times with a minimum interval of 30 seconds, using a mercury sphygmomanometer and Korotkoff sound technique, with an accuracy of $2 \mathrm{~mm} \mathrm{Hg}$, after resting for at least five minutes sitting on chair. Average of two measurements was considered as the final pressure. Systolic blood pressure with the first sound to be heard and diastolic blood pressure with the disappearance of sound was recorded (14).

\subsection{Laboratory Measurements}

All tests were performed in Tehran lipid and glucose research center lab on the same day of sampling. A venous blood sample was obtained from all subjects after 12 - 14 hours of fasting during the night, between 7 - $9 \mathrm{am}$, to measure blood glucose level and lipid levels including triglycerides and HDL-C and was centrifuged in $30-45$ minutes from the time it was collected. Blood glucose was measured on the day of blood collection using the 
enzymatic colorimetric method with glucose oxidase. Serum concentration of triglycerides was measured using commercially available enzymatic reagent (Pars Azmoon, Tehran, Iran) adapted to the Selectra auto analyzer. HDL-C was measured after precipitation of the apolipoprotein $B$ with a solution of phosphotungstic acid. Inter- and assay intra-assay coefficients for all variables were $<5 \%$ (14).

\subsection{Body Mass Index (BMI)}

BMI was calculated by dividing weight by the square of height. Those with BMI less than $85^{\text {th }}$ percentile for the age and sex of Iranian population (based on the CASPIAN study) were considered normal; subjects with a BMI greater or equal to the $85^{\text {th }}$ percentile and below the $95^{\text {th }}$ percentile at risk for overweight and those with BMI greater or equal to the $95^{\text {th }}$ percentile were considered overweight (1).

\subsection{Metabolic Syndrome (MetS)}

In this study, MetS in adolescents was defined based on a modified definition of de Ferranti (20), as having at least three of the following components: fasting plasma glucose concentration greater or equal to $110 \mathrm{mg} / \mathrm{dL}$, fasting serum triglyceride greater or equal to $100 \mathrm{mg} /$ dL, HDL-C less than $45 \mathrm{mg} / \mathrm{dL}$ for boys 15 to 19 years old and less than $50 \mathrm{mg} / \mathrm{dL}$ for other people, WC greater than $75^{\text {th }}$ percentile for the age and sex of Iranian population (based on the CASPIAN study) (21), systolic and diastolic blood pressure greater than $90^{\text {th }}$ percentile for age, sex, and height based on the recommendations of the National Heart, Lung and Blood Institute. WC percentiles for Iranian population has been reported only up to 18 years, hence for individuals older than 18 years high WC was considered based on the criteria of Iranian adults (greater than $95 \mathrm{~cm}$ in both sexes) (22). To as- sess systolic and diastolic blood pressure, on the basis of height-for-age charts of CDC (23), height percentile was determined for each person then using tables published by the National High Blood Pressure Education Program (NHBPEP) for ages 1 to 17 years according to sex, age and height percentile $90^{\text {th }}$ percentile of systolic and diastolic blood pressure were obtained (24). To investigate hypertension in persons over 17 years, NCEP criteria for adults were used (25).

\subsection{Statistical Analysis}

We used SPSS software (version 16; SPSS Inc., Chicago IL) for all statistical analyses. Normal distribution of the data was evaluated by drawing diagram and Kolmogorov-Smirnov test. All components of MetS except triglycerides had normal distribution. To compare quantitative variables between subjects with and without MetS and also between genders, independent t-test and to compare qualitative variables ratio, chi-square test was used and to adjust for confounding variables, analysis of covariance (ANCOVA) was used. Dietary intakes were adjusted for energy by Residual method (26). To calculated the odds ratio (95\% confidence interval) for the MetS and its components in quartiles of total dairy and dairy products intake, logistic regression with adjustment for age, sex, energy intake and BMI was used.

\section{Results}

Mean age and BMI of participants were $14.8 \pm 2.9$ years and $22.1 \pm 4.7 \mathrm{~kg} / \mathrm{m}^{2}$, respectively and $52.9 \%$ were girls. Inter-quartile range of total dairy intake were $<311,311$ - 452, $452-623$ and $\geq 623 \mathrm{~g} / \mathrm{d}$. Demographic characteristics and components of the MetS, in adolescents participating in this study, in both healthy subjects and subjects with MetS are shown in Table 1.

Table 1. Demographic Characteristics and Components of Metabolic Syndrome in Healthy Subjects and Those With MetS a,b

\begin{tabular}{|c|c|c|c|}
\hline $\begin{array}{l}\text { Demographic Characteristics and } \\
\text { Components of MetS }\end{array}$ & Non-MetS Subject $(\mathrm{n}=611)$ & Subjects With MetS $(\mathrm{n}=174)$ & PValue ${ }^{b}$ \\
\hline Age, $y$ & $15.0 \pm 2.9$ & $14.6 \pm 2.9$ & 0.56 \\
\hline Men, \% & 45.3 & 52.8 & 0.02 \\
\hline BMI, $\mathrm{kg} / \mathrm{m}^{2}$ & $21.1 \pm 4.2$ & $25.2 \pm 4.4$ & 0.005 \\
\hline Fasting blood glucose, mg/dL & $91.8 \pm 8.1$ & $94.6 \pm 7.8$ & 0.001 \\
\hline Triglyceride, mg/dL & $74(60-94)$ & $131(113-166)$ & 0.001 \\
\hline HDL cholesterol, mg/dL & $52.5 \pm 10.3$ & $40.9 \pm 6.9$ & 0.001 \\
\hline Waist circumferences, $\mathrm{cm}$ & $74.2 \pm 11.5$ & $86.3 \pm 10.9$ & 0.001 \\
\hline Systolic blood pressure, $\mathrm{mmHg}$ & $100 \pm 11.6$ & $107 \pm 13.0$ & 0.001 \\
\hline Diastolic blood pressure, $\mathrm{mmHg}$ & $66.2 \pm 9.4$ & $70.3 \pm 11.6$ & 0.001 \\
\hline
\end{tabular}

\footnotetext{
a Data are presented as Mean \pm SD except for TG. For TG median and interquartile ranges are reported.

b P values, except for TG, were obtained using t-test and for TG was obtained using Mann-Whitney U test.
} 
MetS was defined according to the de Ferranti criteria. The prevalence of MetS was 22.2\% (19.5\% of girls and $25.2 \%$ of boys). The most prevalent risk factor for MetS among boys was high WC (53.4\%) and among girls was low HDL-C (53.1\%). Dietary intakes of the participants across quartiles of dairy consumption are presented in Table 2. Energy density and intakes of protein, total fat, saturated fat, cholesterol, calcium and phosphor were higher in the highest quartile of dairy consumption compared to the lowest. Prevalence of MetS and its components in quartiles of dairy intake was not significantly different. There were no significant differences in the mean components of MetS among quartiles of dairy intake.
Prevalence of MetS and its components and adjusted mean components of MetS according to quartiles of dairy consumption are shown in Tables 3 and 4, respectively. The odds (95\% CI) for the MetS and its components across quartiles of dairy consumption are shown in Table 5 ; no significant association was observed between total dairy intake and MetS and its components. In Figure 1, the odds $(95 \% \mathrm{CI})$ of MetS across quartile categories of dairy products are illustrated. The odds of Mets, in the highest compared to the lowest quartile of total dairy, low fat dairy, high fat dairy, milk, yoghurt and cheese were 0.97 $(95 \% \mathrm{CI}=0.57-1.66), 1.44$ (95\% CI $=0.83-2.49), 0.97$ (95\% CI $=0.56-1.67), 0.70(95 \% \mathrm{CI}=0.42-1.18), 1.62(95 \% \mathrm{CI}=0.99$ $2.64)$ and $0.72(95 \% \mathrm{CI}=0.44-1.18)$, respectively.

\begin{tabular}{|c|c|c|c|c|c|}
\hline \multirow[t]{2}{*}{ Dietary Intakes } & \multicolumn{4}{|c|}{ Quartile of Dairy Intake } & \multirow[t]{2}{*}{ PValue $^{b}$} \\
\hline & $\mathbf{1}$ & 2 & 3 & 4 & \\
\hline Total energy, kcal/d & $2923 \pm 57.9$ & $2561 \pm 57.6$ & $2545 \pm 57.8$ & $2895 \pm 58.0$ & 0.001 \\
\hline Energy density, kcal/100 g & $82.4 \pm 1.3$ & $88.3 \pm 1.3$ & $92.0 \pm 1.3$ & $96.5 \pm 1.3$ & 0.001 \\
\hline Carbohydrate, \% of total energy & $60.5 \pm 1.0$ & $58.2 \pm 1.0$ & $57.2 \pm 1.0$ & $56.9 \pm 1.0$ & 0.04 \\
\hline Protein, \% of total energy & $13.0 \pm 1.0$ & $13.9 \pm 1.0$ & $14.4 \pm 1.0$ & $17.4 \pm 1.0$ & 0.01 \\
\hline Fat, \% of total energy & $29.6 \pm 2.3$ & $31.3 \pm 2.3$ & $31.2 \pm 2.3$ & $37.4 \pm 2.3$ & 0.08 \\
\hline Saturated fat, \% of total energy & $8.6 \pm 2.3$ & $9.9 \pm 2.3$ & $10.8 \pm 2.3$ & $17.2 \pm 2.3$ & 0.04 \\
\hline Dietary fiber, g/1000 kcal & $19.1 \pm 2.5$ & $18.3 \pm 2.5$ & $16.8 \pm 2.5$ & $20.8 \pm 2.5$ & 0.71 \\
\hline Cholesterol, mg/1000 kcal & $81.5 \pm 3.9$ & $91.5 \pm 3.9$ & $98.8 \pm 3.9$ & $112 \pm 3.9$ & 0.001 \\
\hline Calcium, mg/100o kcal & $430 \pm 10.5$ & $539 \pm 10.5$ & $595 \pm 10.5$ & $743 \pm 10.6$ & 0.001 \\
\hline Phosphorus, mg/1000 kcal & $571 \pm 6.2$ & $631 \pm 6.1$ & $688 \pm 6.1$ & $789 \pm 6.2$ & 0.001 \\
\hline
\end{tabular}

\begin{tabular}{|c|c|c|c|c|c|}
\hline \multirow[t]{2}{*}{ Components of Mets } & \multicolumn{4}{|c|}{ Quartile of Dairy Intake } & \multirow[t]{2}{*}{ P Value } \\
\hline & $\mathbf{1}$ & 2 & 3 & 4 & \\
\hline High blood glucose & 2.0 & 1.0 & 1.5 & 0.5 & 0.57 \\
\hline High triglyceride & 37.2 & 32.5 & 35.7 & 34.2 & 0.78 \\
\hline Low HDL-C & 49.5 & 45.7 & 51.0 & 57.1 & 0.15 \\
\hline High waist circumference & 49.5 & 49.7 & 53.6 & 49.5 & 0.82 \\
\hline Elevated blood pressure & 10.2 & 11.7 & 12.2 & 10.2 & 0.89 \\
\hline Metabolic syndrome & 21.4 & 21.8 & 23.5 & 21.9 & 0.96 \\
\hline
\end{tabular}

\footnotetext{
a Data are percent as percentage. Lowest to highest quartiles of total dairy intake were respectively: $<311.3,311.3-452.5,452.5-622.8,>622.8 \mathrm{~g} / \mathrm{d}$.
} 
Ghotboddin Mohammadi S et al.

Table 4. Adjusted Means for Components of Metabolic Syndrome Across Quartiles of Total Dairy Intake a,b

\begin{tabular}{|c|c|c|c|c|c|}
\hline \multirow[t]{2}{*}{ Components of MetS } & \multicolumn{4}{|c|}{ Quartile of Dairy Intake } & \multirow[t]{2}{*}{ PValue $^{b}$} \\
\hline & 2 & $\mathbf{1}$ & 3 & 4 & \\
\hline Fasting blood glucose, $\mathrm{mg} / \mathrm{dL}$ & $92.3 \pm 0.6$ & $92.3 \pm 0.6$ & $92.6 \pm 0.6$ & $92.5 \pm 0.6$ & 0.96 \\
\hline Triglyceride, mg/dL & $84(65-115)$ & $83(60-109)$ & $84(64-119)$ & $81.5(64-115)$ & 0.41 \\
\hline HDL cholesterol, mg/dL & $50.5 \pm 0.8$ & $50.4 \pm 0.7$ & $49.9 \pm 0.8$ & $48.9 \pm 0.8$ & 0.42 \\
\hline Waist circumference, $\mathrm{cm}$ & $76.5 \pm 0.8$ & $76.5 \pm 0.8$ & $77.5 \pm 0.8$ & $77.0 \pm 0.8$ & 0.77 \\
\hline Systolic blood pressure, $\mathrm{mmHg}$ & $101 \pm 0.8$ & $101 \pm 0.8$ & $102 \pm 0.8$ & $103 \pm 0.9$ & 0.18 \\
\hline Diastolic blood pressure, $\mathrm{mmHg}$ & $66.5 \pm 0.7$ & $66.8 \pm 0.7$ & $68.2 \pm 0.7$ & $67.0 \pm 0.7$ & 0.35 \\
\hline
\end{tabular}

a Data are presented as mean \pm SD except for TG. For TG median and interquartile range are reported. P value was obtained using ANCOVA after adjustment for age, sex and energy intake.

b P value was obtained for TG using Kruskal Wallis test. Lowest to highest quartiles of total dairy intake were respectively: $<311.3,311.3-452.5,452.5$ $622.8,>622.8 \mathrm{~g} / \mathrm{d}$.

Table 5. Multivariate-Adjusted Odds Ratios and 95\% Confidence Interval for Metabolic Syndrome and its Components Across Quartiles of Total Dairy Intake ${ }^{a}$

\begin{tabular}{|c|c|c|c|c|c|}
\hline \multirow{2}{*}{$\begin{array}{l}\text { Metabolic syndrome and its } \\
\text { components }\end{array}$} & \multicolumn{4}{|c|}{ Quartile of Dairy Intake } & \multirow[t]{2}{*}{ PValue } \\
\hline & 1 & 2 & 3 & 4 & \\
\hline \multicolumn{6}{|l|}{ Metabolic syndrome } \\
\hline Model 1 & 1.00 & $1.0(0.62-1.62)$ & $1.06(0.66-1.72)$ & $0.97(0.59-1.58)$ & 0.98 \\
\hline Model 2 & 1.00 & $0.98(0.60-1.60)$ & $1.04(0.64-1.69)$ & $0.97(0.59-1.58)$ & 0.99 \\
\hline Model 3 & 1.00 & $1.15(0.66-1.99)$ & $1.01(0.58-1.74)$ & $0.97(0.59-1.58)$ & 0.93 \\
\hline \multicolumn{6}{|l|}{ High waist circumference } \\
\hline Model 1 & 1.00 & $0.99(0.66-1.47)$ & $1.13(0.76-1.68)$ & $0.94(0.63-1.41)$ & 0.84 \\
\hline Model 2 & 1.00 & $1.01(0.67-1.51)$ & $1.15(0.77-1.73)$ & $0.95(0.63-1.41)$ & 0.80 \\
\hline Model 3 & 1.00 & $1.60(0.88-2.93)$ & $1.30(0.70-2.38)$ & $0.77(0.43-1.40)$ & 0.09 \\
\hline \multicolumn{6}{|l|}{ Low HDL-C } \\
\hline Model 1 & 1.00 & $0.86(0.58-1.28)$ & $1.08(0.72-1.60)$ & $1.37(0.92-2.05)$ & 0.14 \\
\hline Model 2 & 1.00 & $0.91(0.61-1.37)$ & $1.15(0.78-1.72)$ & $1.38(0.92-2.06)$ & 0.20 \\
\hline Model 3 & 1.00 & $0.97(0.64-1.47)$ & $1.13(0.74-1.72)$ & $1.39(0.92-2.10)$ & 0.32 \\
\hline \multicolumn{6}{|l|}{ High triglyceride } \\
\hline Model 1 & 1.00 & $0.81(0.53-1.23)$ & $0.92(0.61-1.40)$ & $0.89(0.58-1.35)$ & 0.80 \\
\hline Model 2 & 1.00 & $0.80(0.52-1.22)$ & $0.91(0.60-1.38)$ & $0.89(0.58-1.35)$ & 0.77 \\
\hline Model 3 & 1.00 & $0.84(0.54-1.30)$ & $0.87(0.56-1.35)$ & $0.86(0.56-1.35)$ & 0.86 \\
\hline \multicolumn{6}{|l|}{ High blood glucose } \\
\hline Model 1 & 1.00 & $0.46(0.08-2.56)$ & $0.70(0.15-3.18)$ & $0.20(0.02-1.86)$ & 0.51 \\
\hline Model 2 & 1.00 & $0.36(0.06-2.03)$ & $0.54(0.12-2.54)$ & $0.20(0.02-1.88)$ & 0.45 \\
\hline Model 3 & 1.00 & $0.37(0.06-2.16)$ & $0.51(0.11-2.41)$ & $0.20(0.02-1.82)$ & 0.45 \\
\hline \multicolumn{6}{|l|}{ Elevated blood pressure } \\
\hline Model 1 & 1.00 & $1.1(0.58-2.10)$ & $1.1(0.58-2.10)$ & $0.83(0.43-1.62)$ & 0.80 \\
\hline Model 2 & 1.00 & $1.1(0.61-2.26)$ & $1.1(0.62-2.28)$ & $0.83(0.42-1.62)$ & 0.68 \\
\hline Model 3 & 1.00 & $1.1(0.66-2.49)$ & $1.2(0.61-2.29)$ & $0.85(0.43-1.68)$ & 0.65 \\
\hline
\end{tabular}




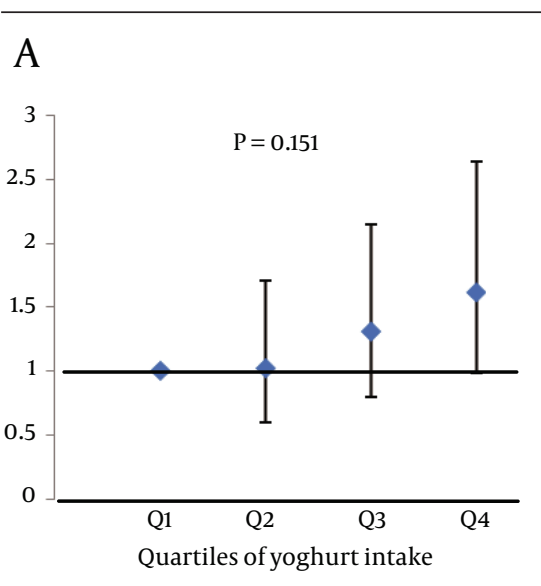

D

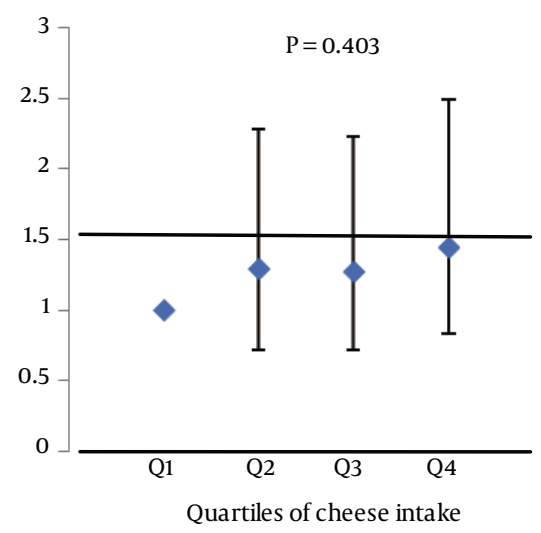

B

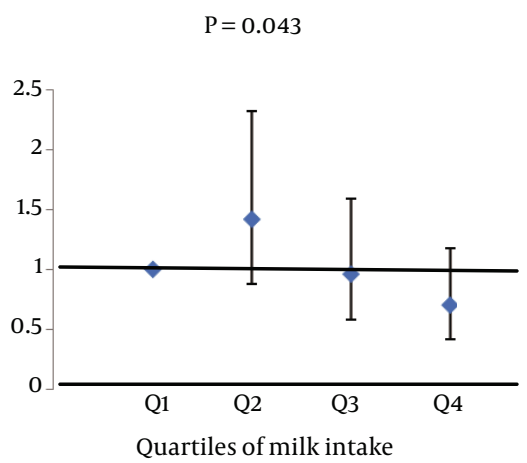

C

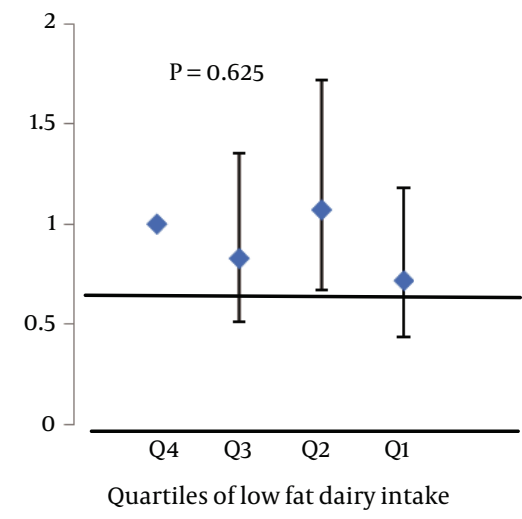

E

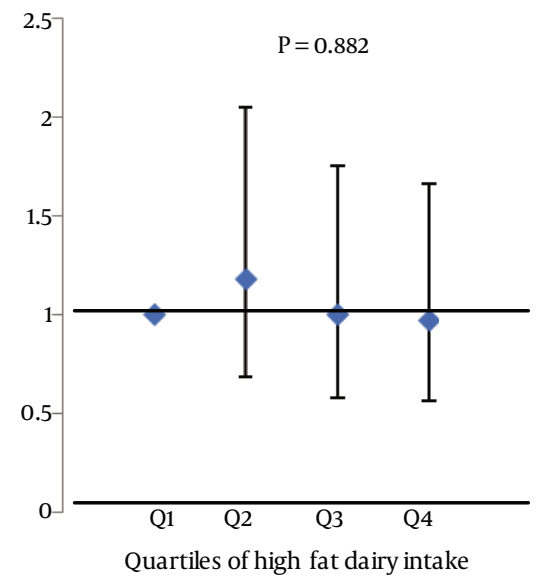

Figure 1. The Odds Ratios With 95\% Confidence Interval for MetS Across Quartiles of Dairy Products

\section{Discussion}

In the present study, based on the definition of de Ferranti, prevalence of MetS was 22.2\% among Tehranian adolescents. However, based on the same definition, the prevalence of MetS in adolescents aged 10 - 18 years has been reported 15.1\% in the first phase of TLGS (2009 - 2011) (27). In this study, after adjusting for confounders, there was no significant association of total dairy intake as well as dairy products with MetS and its components.

Several studies investigated the association between dairy consumption and MetS, although the number of studies on adolescents is limited. A cohort study examined the association of dairy consumption from adolescence to adulthood (13 to 36 years) with incidence of MetS and overweight in adulthood and showed no association between dairy consumption and the incidence of Mets and overweight (13). Results of a cross-sectional study on adolescents aged 15 to 18 years indicated that only milk intake is inversely associated with cardiometabolic risk factor and for the intakes of total dairy, yoghurt and cheese had no significant effect (28). In another study, an inverse association was found between dairy consumption and metabolic syndrome in Tehranian adults aged 18 to 74 years (29).

In this study, there was no significant difference in the odds of high WC, low HDL-C, hypertriglyceridemia, hyperglycemia and hypertension in the highest quartile of total dairy, low-fat and high-fat dairy products and each of dairy products compared to the lowest quartile. The results of studies are inconsistent regarding the association of intake of dairy products and total dairy with overweight, abdominal obesity, dyslipidemia, hyperglycemia and hypertension.

A study on 18 - 30 years old girls showed no significant association of dairy intake with body weight and abdominal obesity (30). Results of a study on children and adolescents indicated that dairy consumption increased the risk of overweight and obesity (31). In contrast, consumption of dairy products $\geq 2$ cups/d was inversely associated with abdominal obesity in boys (32). 
Results of a prospective study on adults showed that subjects with a BMI $<25 \mathrm{~kg} / \mathrm{m}^{2}$ consumed more dairy products; in this study dairy intake was significantly associated with a decreased HDL-C levels (33). In another prospective study, higher intake of dairy products was associated with decreased levels of HDL-C in men (11). The results of a cross-sectional study indicated that yogurt consumption increased HDL-C levels only among women (34). While the results of an 8-week clinical trial showed that low-fat dairy consumption is associated with decreased levels of HDL-C (35). Results from several studies showed that high-fat dairy intake is inversely associated with hypertriglyceridemia (36-38). While several studies have shown no significant association between dairy intake and hypertriglyceridemia (29, 33, 35, 39). A cross-sectional study showed that milk consumption did not cause a change in fasting blood glucose levels while yoghurt consumption reduced and cheese consumption increased fasting blood glucose levels (34). Results from several cross-sectional studies in adults showed that dairy intake is positively associated with fasting blood glucose levels $(37,38)$. Several different studies reported no significant association between dairy consumption and hyperglycemia $(29,36)$. In the present study, there was no association between dairy intake and hyperglycemia, which can be due to low prevalence of patients with hyperglycemia (1.3\%).

Results of a cross-sectional study in adults showed that milk consumption is inversely associated with systolic and diastolic blood pressure. Also yoghurt consumption reduced systolic blood pressure, cheese consumption increased systolic blood pressure (34). In most studies, a significant inverse association was observed between dairy intake and blood pressure in patients with hypertension $(38,39)$. Beneficial effects of dietary calcium on blood pressure in certain populations such as the elderly and African Americans have been more observed than others. This is because of their heightened sensitivity to dietary sodium, which makes them more dependent on calcium release from the extracellular space (40). The findings of this study showed that the risk of hypertension in quartiles of dairy consumption and each of its components is not significantly different; because, the current study was conducted on adolescents and most of them were normotensive. The mechanism of the effect of dairy intake on blood pressure and lipid profile is mainly associated with the effect of whey protein on the activity of angiotensin converting enzyme (41). Several studies suggested that intakes of dairy products and dietary calcium reduce body weight and fat mass and calcium from dairy has more beneficial effects than non-dairy calcium (42). The association between calcium and metabolic syndrome and its components in older populations is stronger because the elderly are more prone to disturbances in calcium metabolism due to vitamin D deficiency (38). The main mechanism of calcium in reducing the risk of MetS and its components is attributed to regulation of calci- trophic hormones, binding with bile acids and increased fat excretion in the feces, regulation of intracellular calcium levels and alteration of metabolism of other electrolytes such as sodium (43).

Several reasons could describe null association of dairy and MetS in this study as well as inconsistent findings of other studies; lower intake of dairy products in this population compared to other population may be a main reason for the findings. Different definitions of MetS in various studies is also another considerable issue. The difference in the age group is another factor for inconsistent results. Often in the elderly population, the association between dairy consumption and MetS was significant. It could be because of their longer exposure to risk factors (including diet) and a greater prevalence of metabolic disturbances (38). Also factors such as the type of dairy product, fat and mineral content may influence the association between dairy intake and MetS (44). Lack of association between dairy consumption and MetS in this study could be due to greater intake of high fat dairy. In other countries, most dairy products fortified with vitamin $\mathrm{D}$, while dairy products in our country are lacking vitamin D (40). Dairy processing generates a variety of biochemical changes to dairy composition, including loss of more labile constituents (such as vitamin C and enzymes), removal of bioactive components such as whey protein from cheese and addition of ingredients such as sugar to yoghurt or salt to cheese (28). An additional factor is known genetic variation in certain ancestral groups (such as African American and East Asian) affecting an individual's ability to digest and absorb components of dairy products. Therefore, ancestry may be a further confounding factor in the relationship between dairy intake and risk of MetS (44).

An important limitation of this study was its cross-sectional design. Due to incomplete Iranian food composition table in the number of food items and micronutrients, for most food items except for items like kashk, USDA food composition table was used. Although the effect of confounders were considered in this study as much as possible, but we were not able to control the effect of genetic factors on MetS. The strength of this study was population-based design. In addition, measurement and control of confounders is another strength of the present study. In general, this study did not support the hypothesis that dairy products consumption protects against MetS and its components.

\section{Acknowledgements}

We thank the TLGS participants and the field investigators of the TLGS for their assistance in physical examinations, biochemical and nutritional evaluation and database management. This study was supported by grant 121 from the National Research Council of Islamic Republic of Iran and the Research Institute for Endocrine Sciences of Shahid Beheshti University of Medical Sciences. The 
authors wish to thank Ms. N. Shiva for critical editinsg of English grammar and syntax.

\section{Authors' Contributions}

The project idea for this study was from shirin ghotboddin Mohammadi. The project was design by Shirin Ghotboddin Mohammadi, Parvin Mirmiran, Zahra bahadoran. Yadollah Mehrabi, Shirin Ghotboddin Mohammadi, Zahra bahadoran analyzed and interpreted the data. Shirin Ghotboddin Mohammadi, Zahra Bahadoran, Parvin Mirmiran and Fereidoun Azizi prepared the manuscript. All authors read and approved the final version of the manuscript for submission.

\section{Funding/Support}

This study was supported in part by grant 750 from the research institute for endocrine sciences, Shahid Beheshti University of Medical Sciences, Tehran, Iran.

\section{References}

1. Kelishadi R, Ardalan G, Gheiratmand R, Majdzadeh R, Hosseini M, Gouya MM, et al. Thinness, overweight and obesity in a national sample of Iranian children and adolescents: CASPIAN Study. Child Care Health Dev. 2008;34(1):44-54.

2. Lutsey PL, Steffen LM, Stevens J. Dietary intake and the development of the metabolic syndrome: the Atherosclerosis Risk in Communities study. Circulation. 2008;117(6):754-61.

3. Azizi F, Salehi P, Etemadi A, Zahedi-Asl S. Prevalence of metabolic syndrome in an urban population: Tehran Lipid and Glucose Study. Diabetes Res Clin Pract. 2003;61(1):29-37.

4. Esmaillzadeh A, Mirmiran P, Azadbakht L, Etemadi A, Azizi F. High prevalence of the metabolic syndrome in Iranian adolescents. Obesity (Silver Spring). 2006;14(3):377-82.

5. Cook S, Auinger P, Li C, Ford ES. Metabolic syndrome rates in United States adolescents, from the National Health and Nutrition Examination Survey, 1999-20 02. JPediatr. 2008;152(2):165-70.

6. Hadad Tabrizi S, Haji Faraji M, Houshiar Rad A, Abadi A, Hosseinpanah F. Association between the metabolic syndrome and food patterns in non-menopause women. Iranian Journal of Nutrition Sciences \& Food Technology. 2010;5(1):39-48.

7. Noori N, Mirmiran P, Asgari S, Azizi F. Dietary Intake of Calcium and Vitamin D and the Prevalence of Metabolic Syndrome in Tehranian Adults: Tehran Lipid and Glucose Study (TLGS). Iranian Journal of Endocrinology and Metabolism. 2007;9(2):191-200.

8. Wang H, Steffen LM, Vessby B, Basu S, Steinberger J, Moran A, et al. Obesity modifies the relations between serum markers of dairy fats and inflammation and oxidative stress among adolescents. Obesity (Silver Spring). 2011;19(12):2404-10.

9. Nestel PJ. Effects of dairy fats within different foods on plasma lipids. JAm Coll Nutr. 2008;27(6):735S-40S.

10. Ruidavets JB, Bongard V, Dallongeville J, Arveiler D, Ducimetiere P, Perret B, et al. High consumptions of grain, fish, dairy products and combinations of these are associated with a low prevalence of metabolic syndrome. J Epidemiol Community Health. 2007;61(9):810-7.

11. Fumeron F, Lamri A, Abi Khalil C, Jaziri R, Porchay-Balderelli I, Lantieri O, et al. Dairy consumption and the incidence of hyperglycemia and the metabolic syndrome: results from a french prospective study, Data from the Epidemiological Study on the Insulin Resistance Syndrome (DESIR). Diabetes Care. 2011;34(4):813-7.

12. Lawlor DA, Ebrahim S, Timpson N, Davey Smith G. Avoiding milk is associated with a reduced risk of insulin resistance and the metabolic syndrome: findings from the British Women's Heart and Health Study. Diabet Med. 2005;22(6):808-11

13. te Velde SJ, Snijder MB, van Dijk AE, Brug J, Koppes LL, van Mech- elen W, et al. Dairy intake from adolescence into adulthood is not associated with being overweight and metabolic syndrome in adulthood: the Amsterdam Growth and Health Longitudinal Study. J Hum Nutr Diet. 2011;24(3):233-44.

14. Azizi F, Ghanbarian A, Momenan AA, Hadaegh F, Mirmiran P, Hedayati M, et al. Prevention of non-communicable disease in a population in nutrition transition: Tehran Lipid and Glucose Study phase II. Trials. 2009;10:5.

15. Willett W. Issues in Analysis and Presentation of Dietary Data. Nutritional epidemiology.1998;2:321-46.

16. Ghaffarpour M, Houshiar-Rad A, Kianfar H. [The manual for household measures, cooking yields factors and edible portion of foods].Tehran: Nashre Olume Keshavarzy; 1999.

17. Mirmiran P, Esfahani FH, Mehrabi Y, Hedayati M, Azizi F. Reliability and relative validity of an FFQ for nutrients in the Tehran lipid and glucose study. Public Health Nutr. 2010;13(5):654-62.

18. Azar M, Sarkisian E. Food composition table of Iran.Tehran: National Nutrition and Food Research Institute, Shaheed Beheshti University; 1980

19. USDA. USDA national nutrient database for standard reference, release 21. In: US Department of Agriculture, editor. united states: 2011.

20. de Ferranti SD, Gauvreau K, Ludwig DS, Neufeld EJ, Newburger JW, Rifai N. Prevalence of the metabolic syndrome in American adolescents: findings from the Third National Health and Nutrition Examination Survey. Circulation. 2004;110(16):2494-7.

21. Kelishadi R, Gouya MM, Ardalan G, Hosseini M, Motaghian M, Delavari A, et al. First reference curves of waist and hip circumferences in an Asian population of youths: CASPIAN study. J Trop Pediatr. 2007;53(3):158-64.

22. Azizi F, Khalili D, Aghajani H, Esteghamati A, Hosseinpanah F, Delavari A, et al. Appropriate waist circumference cut-off points among Iranian adults: the first report of the Iranian National Committee of Obesity. Arch Iran Med. 2010;13(3):243-4.

23. CDC. Data Table of Stature-for-age Charts. 2012. Available from http://www.cdc.gov/growthcharts/htmlcharts/statge.htm.

24. National High Blood Pressure Education Program Working Group on High Blood Pressure in C, Adolescents. The fourth report on the diagnosis, evaluation, and treatment of high blood pressure in children and adolescents. Pediatrics. 2004;114(2 Suppl 4th Report):555-76.

25. Grundy SM, Cleeman JI, Daniels SR, Donato KA, Eckel RH, Franklin $\mathrm{BA}$, et al. Diagnosis and management of the metabolic syndrome: an American Heart Association/National Heart, Lung, and Blood Institute Scientific Statement. Circulation. 2005;112(17):2735-52.

26. Willett WC, Howe GR, Kushi LH. Adjustment for total energy intake in epidemiologic studies. Am J Clin Nutr. 1997;65(4 Suppl):1220S-8S.

27. Hosseini-Esfahani F, Mousavi Nasl Khameneh A, Mirmiran P, Ghanbarian A, Azizi F. Trends in risk factors for cardiovascular disease among Iranian adolescents: the Tehran Lipid and Glucose Study, 1999-2008. J Epidemiol. 2011;21(5):319-28.

28. Abreu S, Moreira P, Moreira C, Mota J, Moreira-Silva I, Santos PC et al. Intake of milk, but not total dairy, yogurt, or cheese, is negatively associated with the clustering of cardiometabolic risk factors in adolescents. Nutr Res. 2014;34(1):48-57.

29. Azadbakht L, Mirmiran P, Esmaillzadeh A, Azizi F. Dairy consumption is inversely associated with the prevalence of the metabolic syndrome in Tehranian adults. Am J Clin Nutr. 2005;82(3):523-30.

30. Bank SS, Ghanjali N, Ghalaeh RS, Azadbakht L. Association between dairy and calcium intake and general and central obesity among female students. J Educ Health Promot. 2013;2:16.

31. Matthews VL, Wien M, Sabate J. The risk of child and adolescent overweight is related to types of food consumed. NutrJ. 2011;10:71.

32. Abreu S, Santos R, Moreira C, Vale S, Santos PC, Soares-Miranda L, et al. Association between dairy product intake and abdominal obesity in Azorean adolescents. Eur J Clin Nutr. 2012;66(7):830-5.

33. Snijder MB, van Dam RM, Stehouwer CD, Hiddink GJ, Heine RJ Dekker JM. A prospective study of dairy consumption in relation to changes in metabolic risk factors: the Hoorn Study. Obesity (Silver Spring). 2008;16(3):706-9.

34. Beydoun MA, Gary TL, Caballero BH, Lawrence RS, Cheskin LJ, 
Wang Y. Ethnic differences in dairy and related nutrient consumption among US adults and their association with obesity, central obesity, and the metabolic syndrome. The American journal of clinical nutrition. 2008;87(6):1914-25.

35. van Meijl LE, Mensink RP. Low-fat dairy consumption reduces systolic blood pressure, but does not improve other metabolic risk parameters in overweight and obese subjects. Nutr Metab Cardiovasc Dis. 2011;21(5):355-61.

36. Duffey KJ, Gordon-Larsen P, Steffen LM, Jacobs DR, Popkin BM. Drinking caloric beverages increases the risk of adverse cardiometabolic outcomes in the Coronary Artery Risk Development in Young Adults (CARDIA) Study. American Journal of Clinical Nutrition. 2010;92(4):954-9.

37. Sun Y, Jiang C, Cheng KK, Zhang W, Leung GM, Lam TH, et al. Milk consumption and cardiovascular risk factors in older Chinese: the Guangzhou Biobank Cohort Study. PLoS One. 2014;9(1):e84813.

38. Snijder MB, van der Heijden AAWA, van Dam RM, Stehouwer CDA Hiddink GJ, Nijpels G, et al. Is higher dairy consumption associated with lower body weight and fewer metabolic disturbances? The Hoorn Study. The American journal of clinical nutrition.
2007;85(4):989-95.

39. Wennersberg MH, Smedman A, Turpeinen AM, Retterstol K, Tengblad S, Lipre E, et al. Dairy products and metabolic effects in overweight men and women: results from a 6-mo intervention study. Am J Clin Nutr. 2009;90(4):960-8.

40. Rice BH, Cifelli CJ, Pikosky MA, Miller GD. Dairy components and risk factors for cardiometabolic syndrome: recent evidence and opportunities for future research. Adv Nutr. 2011;2(5):396-407.

41. FitzGerald RJ, Meisel H. Milk protein-derived peptide inhibitors of angiotensin-I-converting enzyme. Br J Nutr. 2000;84 Suppl 1:S33-7.

42. Zemel MB, Thompson W, Milstead A, Morris K, Campbell P. Calcium and dairy acceleration of weight and fat loss during energy restriction in obese adults. Obes Res. 2004;12(4):582-90.

43. Zemel MB. Mechanisms of dairy modulation of adiposity. J Nutr. 2003;133(1):252S-6S

44. Baxter AJ, Coyne T, McClintock C. Dietary patterns and metabolic syndrome--a review of epidemiologic evidence. Asia Pac J Clin Nutr. 2006;15(2):134-42. 